his pen have appeared in the Botanische zeitung, Bienen zeitung, Kosmos, Nature, etc., while, as editor of the department of Justs' Jahresbericht, relating to pollination and dissemination, he has contributed reviews of all of the more important publications bearing on his specialty. Beside these, he published two books, - Befruchtung der blumen durch insekten, und die gegenseitigen anpassungen beider (which appeared in 1873 , served as the basis of a very instructive series of articles in Nature, and was largely drawn upon by Lubbock in the preparation of his little work on British wild-flowers, and which, supplemented by the more recent observations of its author, has lately been translated into English); and Alpenblumen, ihre befruchtung durch insekten und ihre anpassungen an dieselben (a volume of equal size, published in 1881, and, like its predecessor, filled with instructive facts).

From the first, Dr. Müller was a pronounced evolutionist, perhaps erring in too exclusive contemplation of a limited part of the evidence of derivation, and, like many others of the German school, inclined to push evolutionary logic to its ultimate if undemonstrable conclusion of materialism.

As a teacher he was most excellent, having the faculty, not only of imparting ideas to his pupils, but of inspiring their enthusiasm. In his specialty he was a careful observer, noting and accounting for many minute structural peculiarities in both flower's and insects, which, so long as their utility remained undiscovered, were explicable only by the theory of types in nature. So far as observation is concerned, his work is above criticism. As a rule, too, his inferences are correctly drawn, though the limitation of his studies to a small part of the world has at times rendered his enthusiasm over the biological significance of some supposed new adaptation, subject to the criticism of specialists previously familiar with the structure, if not with its meaning.

As a friend, Dr. Müller was always cordial, ever ready with encouragement and assistance for younger workers in the line of his specialty.
He had, however, little patience with inaccuracy in observation, and, both publicly and in private, criticised errors with vigor; but, though his criticisms were sometimes severe, they were seldom unkind, and never unjust. By his death, biological science loses not only one of its most enthusiastic and able devotees, but also one, who, by the independent and thorough nature of his work, may be styled not inappropriately an epoch-maker.

\section{THE USE OF THE SPECTROSCOPE IN METEOROLOGY.}

In April last it was thought desirable to add to the regular meteorological observation made at the Shattuck observatory, Dartmouth college, the hygrometric indications of the spectroscope. The observations were made in accordance with the directions of J. Rand Capron in his 'Plea for the rain-band.' The instruments used were two direct vision spectroscopes: one a $3 \frac{1}{2}$-inch 'vest-pocket' instrument of Hofmann's; the other 10 inches in length, and capable of separating the $D$ lines with direct sunlight. The observations made in this way were found to be interesting, but unsatisfactory. The difficulty which an observer must always find in estimating confidently the degree of intensity of the absorption lines and bands with the widely varying lights of fair and cloudy weather, makes the arrangement of some method of measurement very desirable. After a few trials in other directions, the device described below was decided upon, and has proved satisfactory. It was thought that the absorption lines of aqueous vapor, seen with a spectroscope of rather high power, are better adapted to delicate measurement than the broad band seen with a low power. The small spectroscope used shows the dark band on the red side of the $D$ line with great clearness; but the absorption lines are only visible when particularly strong. With the larger instrument, however, the spectrum is so elongated that the general darkening near $D$ is hardly noticeable; while the two moisture lines to be found there are very prominent. The apparatus illustrated is designed to measure the variation in intensity of the darker line of this pair (the $\alpha$ of the $D$ group of Janssen's map).

The only methods of measurement of the intensity of absorption lines, known to the writer, are those of Janssen and Gouy. The 
former, in 1871, in his work in mapping the atmosphere lines, used for comparison black lines of various widths, ruled on white paper, and viewed through vessels filled with darkened water. ${ }^{1}$ Gouy made some measurements of solar lines by photometric methods; isolating a narrow strip of the spectrum adjacent to the line, and comparing its light with that of a strip of equal width containing the line. From these data he calculated the intensity of the line, not in photometric, but in linear units. ${ }^{2}$ The method adopted by the writer is entirely different from either of these ; and, as far as known, is new.

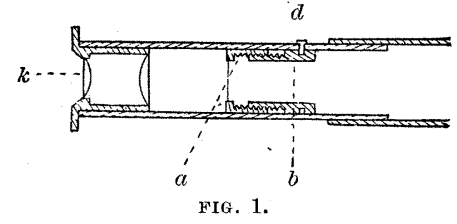

What was desired was the production of an artificial absiorption spectrum, the intensity of whose lines could be varied at will, until one of the lines thus produced should be sensibly the same as the line to be measured. Fig. 1 is a section of the attachment to the spectroscope made for this purpose. The dark lines required are diffraction fringes produced at the focus of the positive eye-piece, which are therefore seen projected on the spectrum. They are produced by placing a silk fibre a little beyond the focus of the eyepiece. In the figure, the piece $a$ slides in the tube, bearing with it a single silk fibre placed vertically and just in the middle of the field of view of the eye-piece. The fibre is maintained vertical by means of a projecting pin sliding in a longitudinal slot in the tube, as shown at $s$ in fig. 2. The sliding motion is given to it by means of the piece $b$, which turns freely, but cannot slide, being retained by the screw $d$ fitting in a groove made entirely around the piece. Two openings are made in the tube, on opposite sides, so that $b$ can be turned directly with the fingers. One of these windows is shown at $n$ in fig. 2. By turning in one direction, the silk fibre may be put nearly in the focus : by turning back, it can be made invisible. When near the focus, the fibre appears as a pair of dark parallel lines and quite close together. As it is drawn away from the focus, the lines appear to separate somewhat, growing constantly fainter until they disappear. The fainter diffraction fringes produced are invisible in the rather weak light of the spectrum. Whole revolutions of the screw $b$ are read off on the graduation at the side of the slot, and fractions (tenths) are read from the piece itself, which is graduated as a micrometer screw. The lines thus produced resemble closely the $D$ group, particularly when both are strong, when a very sharp eye is required to distinguish the spurious lines from the genuine. As the movement of the eye from side to side would modify the appearance of the interference lines, making one darker than the other, the spectrum must be viewed through a narrow vertical opening, making such motion impossible. For this purpose a piece of black paper (not shown in the figure), provided with a vertical slit of perhaps $0.7 \mathrm{~mm}$. width, must be placed on the eye-lens at $k$. Even with this, a little care is necessary in the position of the eye, that the pair of lines shall always be equal. The slight darkening of the spectrum between the two lines, which occurs, is in this case not objectionable, as it imitates pretty closely the general absorption in the space between $D$ and the $\alpha$ line of the $D$ group. The instrument, as figured, is provided with a tangent screw at $e$, by which the whole tube containing the eye-piece can be moved horizontally, thus shifting the field of view so that any line of the spectrum can be brought to the side of the comparison lines. The instrument is mounted on a wooden base, grooved at the top to receive it. At the back side is a large knob by which the instrument is held when taking an observation. When directed to any

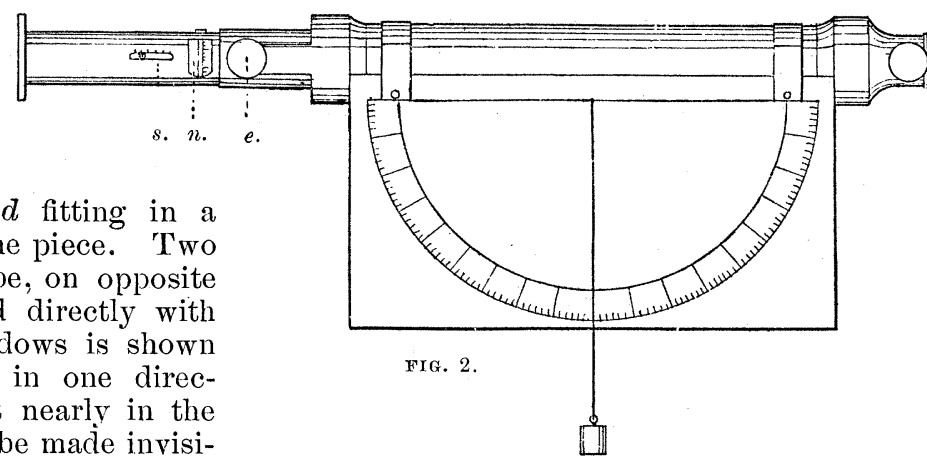

part of the sky, the altitude can be determined by means of the graduated circle and hanging weight shown in the figure.

Another device, much simpler, and of use, 
it is believed, for general observation when less accuracy is required, is shown in fig. 3. A collar represented in section at $a$ is inserted into the tube of the spectroscope, and fastened permanently so that its front side shall be just in the focus of the eye-piece. From the lower front edge to the upper back edge, a silk fibre passes, drawing back as it rises. The fibre will evidently appear in the field of view, as represented at $b$, as lines of diminishing intensity. A set of horizontal, equidistant spider-lines are attached to the front edge, hence.just in focus. 'The

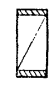

$a$

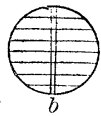

FIG. 3. line whose intensity is to be measured is made to appear parallel and near to one of the interference lines; and its intensity is expressed by the number of the spider-line at which the intensities correspond; counting downwards. And here it may be mentioned that such a scale of intensities (or, indeed, the scale afforded by the micrometer screw readings in the preceding apparatuis) is not a scale of equal parts, a change of a unit in case of a line of high intensity being more than in case of a low intensity. 'This is, however, believed not to be a serious disadvantage in practice.

The advantages of any practicable method of measurement over a mere estimation are evident enough. When estimated by the eye, it is believed to be impracticable to distinguish more than five grades of strength, while by this method quite fine shades of intensity can be measured ; and what is, perhaps, of equal importance, measurements made against dark and light sky are apparently identical, a change in the brilliancy of the background affecting the appearance equally of the absorption and interference lines. Evidently an unaided estimation would very likely be at fault in such a case.

As to the accuracy actually attained in practice, it is found, in looking over the record of about a month past, that the whole range of the readings made at one observation, in ordinarily favorable weather, averages 0.3 of a revolution of the micrometer screw ; and, as from four to twelve or more readings are always taken, according to the aniount of variation noted, the probable error of the mean may be considered as about 0.03 , as computation has shown in a number of cases. Now, as the whole range of the instrument used is from 1.0 to 5.7 , it is evident that many grades of intensity are capable of appreciation. It is to be remembered, that these readings are purposely made in various quarters of the sky, so that discrepancies in readings are partly due to want of uniformity in the hygrometric state of the atmosphere. It should be stated, also, that such accuracy is not attainable below 2.0, as the value of a unit is then considerably less than above that value.

The regular record made at the observatory is as follows: 'The ordinary meteorological record is made three times daily. With the spectroscope, at least three sets of readings are taken, comprising measurements of the intensity of the moisture line at the horizon, at altitudes of $10^{\circ}, 20^{\circ}, 30^{\circ}$, and $90^{\circ}$. In all cases, the readings are taken in all quarters of the sky where there is sufficient light. A set of readings is also taken by setting the micrometer at 2.0, giving a faint line just visible in dark weather, and then measuring the altitude at which the moisture line is of the same strength. Such readings of altitude rarely vary more than $2^{\circ}$ to $4^{\circ}$ in settled weather. The strength of line and of the 'rain-band' is also estimated by the eye at each observation. At the same time the readings of the wet and dry bulb hygrometer are taken, as well as of a Regnault's condensing hygrometer. The wet and dry bulb hygrometer can be ventilated by means of a bellows, as suggested by $\mathrm{Mr}$. H. A. Hazen, in a recent number of Science. Notes are made of the direction and velocity of the wind, of the clouds, and condition of the air.

One of the most interesting of the powers of the spectroscope thus used is its ability to detect relatively moist tracts in the atmosphere. While in settled weather entire uniformity at all points of the horizon is generally noted, in unsettled weather considerable differences are often observed. An excellent example of this power of the instrument occurred on May 26. During the morning when the observations were made, the air was very clear and dry, the moisture line therefore weak. At 6 A.м., measurements made entirely around the horizon showed that the line became invisible very uniformly at an altitude of $10^{\circ}$, except for about $45^{\circ}$ of the north-eastern horizon, where the altitude of disappearance was $20^{\circ}$, while the intensity of the line at the horizon here was about double that elsewhere. There was no wind blowing, and no clouds of any kind were visible except a few wisps of cirrus clond high in the east. 'These facts were all noted in the record at the time. At seven o'clock, when the next readings were taken, to my surprise this moist tract was found to be nearly filled up with a bank of stratus cloud, with no other clouds visible. At the same 
time a similar moist region, of $15^{\circ}$ altitude and perhaps $35^{\circ}$ length, was discovered in the southeast; and this in turn was found fifteen minutes later to be partly filled with cloud. After an hour or so they had all disappeared. The appearance was as though a body of air heavily. charged with moisture, having become heated, was seen rising bodily at six o'clock, while at seren the consequent cooling had condensed in part its moisture.

One of the most striking facts noted is the suddenness with which a hygrometric change occurs, as indicated by the spectroscope. During the fine weather of June 30 and July 1 , the spectroscope had indicated unusually dry air with almost absolute uniformity. During July 1, as the diagram shows, there had been a very slight increase in the moisture present, as indicated by an observation at six P.M. Fifteen minutes later, happening to glance through the spectroscope, I was greatly surprised to see how much blacker the line looked. A new set of readings was taken, giving a much higher amount of moisture, as indicated by the sudden rise in the curve. The sky was almost entirely free from clouds, with a light breeze from the south - west. Measurements were made in both cases all along the western half of the horizon, the eastern being too dark at that hour. At seven o'clock a moderately dense bank of stratus clouds had risen in the west to an altitude of $15^{\circ}$ or $20^{\circ}$. The record at seven and seventhirty showed little further hygrometric change. The sky was soon entirely overcast with clouds. This hygrometric change was not a mere momentary one, connected with cloud-formation ; but the later record showed it to be the beginning of a period of moist air and showery weather. The hygrometer, it will be noted in the diagram, gave little sign of change for some hours. Other sudden changes of equally striking character have been observed. 'That, as has been suggested by Capron and others, the physical state of the suspended water, the size of the aqueous particles, may have an influence in its light-absorbing power, and so explain in part such changes, is very possible; but the evidence that such is the case appears to be far from conclusive.

It is believed that a series of spectroscopic observations, continued for a considerable period of time at different stations, would give much light on a number of important questions in meteorology, particularly in the study of the formation of showers and storms. The instrument is apparently admirably adapted to do this work, by its ability to trace accurately the motions of masses of vapor in the upper atmosphere. The discussion of the more important questions which arise in carry-

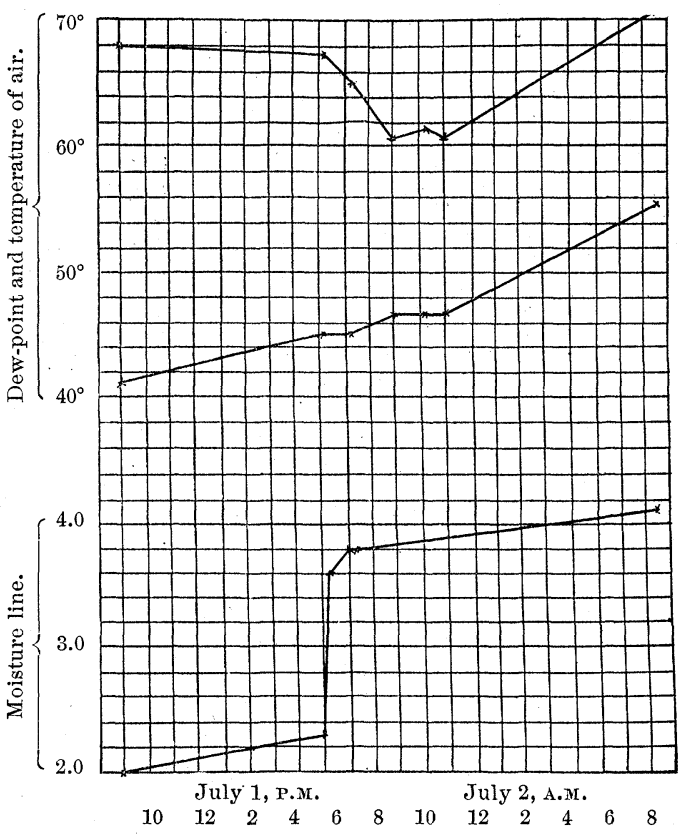

ing on this investigation is deferred until a larger mass of figures and facts have been accumulated.

C. S. Cook.

\section{NOTES ON SASSAFRAS-LEAVES.}

There are three distinct forms of sassafrasleaves. The simplest is ovate, varying to oval and obovate. A second form is threelobed, the incisions running from near the middle of the upper half of the leaf's edge to the centre of the blade. The third form is midway between the entire and three-lobed sorts, and has but one side-lobe; the opposite half of the leaf being entire. It is as if onehalf of a three-lobed leaf were joined by the midrib to the opposite half of an entire one of the same size. This form may be very appropriately called the ' mitten.'

In the study of these three forms, branches of sassafras have been gathered from a large number of places through the surrounding country. Some have been obtained from the woods, and others from the open field. Branches were cut from the largest trees and from the smallest, from vigorous trees and those of slow growth. Ten hundred and fifty leaves were examined : and of these, five hundred and thirteen were entire; four hundred 